\title{
Screening for prostate cancer: Can randomized studies optimize the trade-off between over - and under-diagnosis?
}

\author{
Sandblom Gabriel \\ Department of Surgery, Karolinska University Hospital Huddinge, 14186 Stockholm, Sweden \\ Correspondence: Sandblom Gabriel. Address: Department of Surgery, Karolinska University Hospital Huddinge, 14186 \\ Stockholm, Sweden. Telephone: 46-704-158-218. Fax: 46-858-586-936. E-mail: gabriel.sandblom@ki.se \\ Received: February 7, 2012 \\ Accepted: February 22, $2012 \quad$ Published: April 1, 2012 \\ DOI : $10.5430 /$ jst.v2n2p23 \\ URL: http://dx.doi.org/10.5430/jst.v2n2p23

\begin{abstract}
Objectives: The outcome from the screening trials on prostate cancer published in recent years may at a first glance seem contradictory. A review of all randomized controlled trials was undertaken in order to explore these divergences.

Methods: PubMed was searched for all publications on prostate cancer screening trials.

Results: Five randomized controlled trials were found. The only trial that could show a statistically significant impact on prostate cancer mortality was the European Randomized Study of Screening for Prostate Cancer (ERSPC), in particular the Gothenburg Centre, which constitutes on of eight units of the ERSPC trials. The absence of significant outcome from the other trials may, however, be explained by higher PSA threshold, longer screening intervals and shorter follow-up. Conclusions: Screening for prostate cancer may reduce prostate cancer mortality, but to the cost of over-detection and over-treatment.
\end{abstract}

\section{Key words}

Prostate cancer, Screening, Overdiagnosis, Mortality, PSA

\section{Introduction}

Increasing evidence of improved survival in men with localized prostate cancer treated with radical prostatectomy ${ }^{[1]}$ fuelled the debate on prostate cancer screening at the beginning of the 2000's. Two large randomized controlled trials ${ }^{[2,3]}$ were initiated at the beginning of the 1990's in order to provide ultimate answers to all of the issues regarding prostate cancer screening, in particular: "Does screening save lives or not?". The two studies, however, ended up in a tie-break, with the Prostate, Lung, Colorectal and Ovarian (PLCO) cancer screening trial unable to show any survival benefit and the European Randomized Study of Screening for Prostate Cancer (ERSPC) showing a small, but significant benefit. In addition to the ERSPC and PLCO trials, three minor trials have also provided data regarding screening ${ }^{[4-6]}$, albeit not unequivocally in favor for or against screening. After a period of ambivalence, the US preventive services task force (USPTF) decided to take a standpoint against screening, issuing a recommendation statement concluding that the harms of prostate cancer screening outweigh the benefits ${ }^{[7]}$. 
The greatest problem when interpreting the seemingly contradictory outcomes of prostate cancer trials is not divergence of outcome, but rather the adequacy of the question posed. Instead of asking "Benefit or no benefit?", the question should be "Benefit, but at what cost?” or “Over-detection and over-treatment versus death from prostate cancer”. When approaching the issues of prostate cancer in this manner, the problem is much greater than can ever be solved with a single P-value discriminating between death rates in a screened and a non-screened population. Over-treatment of men with non-lethal tumors is a huge ethical dilemma that should not be neglected. Whereas tumor remission after surgery or radiotherapy may be perceived as confirmation of the benefits of screening with early diagnosis and treatment, the harms of treatment, including perioperative complications, erectile dysfunction and incontinence should not be neglected. Furthermore, despite the fact that the outcome of surgery or radiotherapy may seem successful for men with low-risk tumors, i.e. those usually detected by screening, these men benefit less from treatment with radical intent than do men with high-risk tumors, even though men with high-risk tumors face a higher risk of early tumor recurrence ${ }^{[8]}$. In fact, even the ERSPC showed that 48 men must undergo treatment with curative intent in order for one man to avoid death from prostate cancer ${ }^{[3]}$. On the other hand, preventing death from prostate cancer may be considered important not only for prolonging survival, but also for avoiding a very dreadful period prior to death. Death from prostate cancer may be very agonizing, with constant pain, loss of autonomy, and dependence on help with almost every daily activity ${ }^{[9]}$. During the final year of life, a rapid decrease in perceived quality of life is seen for men with prostate cancer ${ }^{[10]}$.

Ultimately, it is only men with a suspicion of prostate cancer who are able to make an adequate trade-off between the potential hazards of over-diagnosis and the risk of neglecting a potentially curable prostate cancer. This requires informed decision-making, which may be a very complex process that is not easily addressed using the rather schematic routines of a mass screening program. A study has been initiated in Australia with the aim of quantifying men's preferences in a structured way ${ }^{[11]}$. This study may provide a better understanding of the relative importance of the factors influencing the decision to undergo PSA testing.

The aim of the present review was to critically assess and compare the outcomes of the randomized controlled trials presenting mortality data following prostate cancer screening.

\section{Results}

Five randomized studies have presented results on prostate cancer mortality. Although somewhat contradictory, they have each independently shed some light on the outcome of prostate cancer screening:

\section{The prostate, lung, colorectal and ovarian ( PLCO) cancer screening trial} In the first report from the PLCO trial, no survival benefit from screening was seen ${ }^{[2]}$. The lack of survival benefit may, however, have been explained by a relatively high PSA threshold $(4.0 \mathrm{ng} / \mathrm{mL})$, and high pre-screening and contamination levels in the control group. Furthermore, the follow-up was not sufficient to achieve the power to show any differences in mortality between the screening group and control group. In a recent follow-up, a small but non-significant reduction in prostate cancer mortality was seen in the screening group ${ }^{[12]}$. With a long enough follow-up period, this difference between the control group and screening group could become significant.

\section{The European randomized study of screening for prostate cancer (ERSPC)}

The ERSPC was a study organized by assembling data from several countries in Europe. The study design differed slightly between units, but the total study population size was more than twice the size of the PLCO trial. In the first report on prostate cancer mortality from the study, a reduction in prostate cancer mortality of $20 \%(P=0.04)$ was seen ${ }^{[3]}$. The outcome from the ERSPC trial, however, was to a great extent dependent on one center, Gothenburg, where the patients were younger, the PSA threshold lower, the screening intervals shorter and the follow-up longer. In a separate analysis 
from Gothenburg, a reduction in prostate cancer mortality of $44 \%$ was seen ${ }^{[13]}$ which indicates that the favorable outcome of prostate cancer screening seen in the ERSPC trial was, to a great extent, dependent on the Gothenburg subgroup.

\section{The Stockholm study}

In a study performed in Stockholm, Sweden, 2400 men aged 55 to 70 years were invited once to partake in prostate cancer screening that included digital rectal examination, transrectal ultrasound and PSA testing ${ }^{[4]}$. In a 15-year follow-up, no reduction in the overall or prostate cancer-specific survival was seen. Screening subjects, however, had a significantly lower risk for death from causes other than prostate cancer, which raises the possibility of co-morbidity amongst men who did not accept the screening invitation. This is an important observation that must be taken into account when assessing the outcome of screening trials.

\section{The Quebec study}

The first study to ever provide results regarding prostate cancer screening was the Quebec study ${ }^{[5]}$. The original results indicated a great benefit from screening, with a relative prostate cancer death risk in the screened group of 0.38 (95\% confidence interval $0.20-0.73$ ). The results from this study, however, were compromised by a large crossover and contamination between the study group and the control group. When tested in an intention-to-treat analysis, no significant decrease in prostate cancer mortality was seen. Considering the risk for confounding due to high co-morbidity associated with non-participation in screening, as seen in the Stockholm study ${ }^{[4]}$, the outcome of the Quebec study may be very misleading if interpreted inadequately.

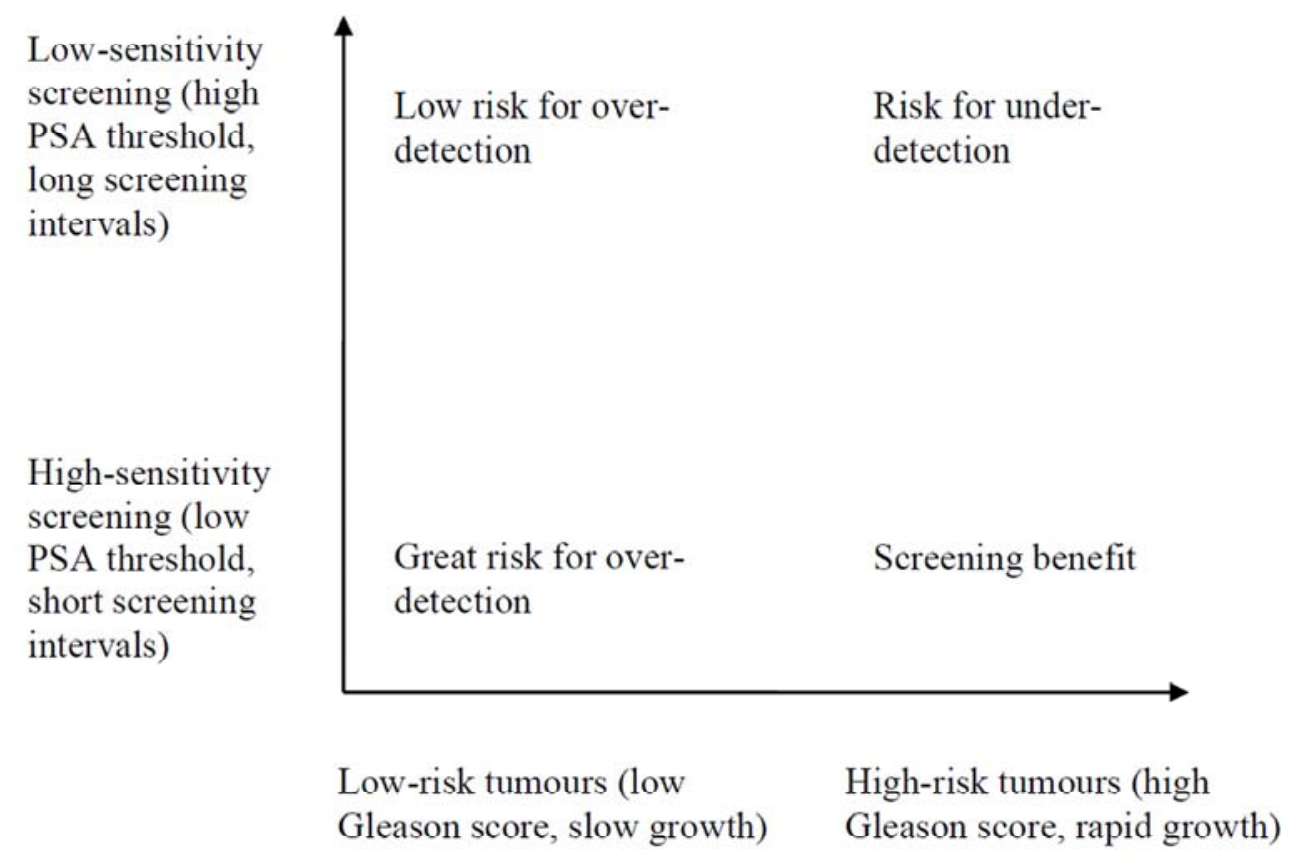

Figure 1. The design of a screening program requires careful consideration of the risk for over-as well as under-detection. Ideally, the most aggressive cancer should constitute a high proportion of the screening-detected tumors ${ }^{[14]}$. However, the high-risk tumors usually grow more rapidly than the low-risk tumors and present as interval cancers with locally advanced growth at diagnosis. 


\section{The Norrkoping trial}

The Norrkoping trial was the first randomised controlled trial on prostate cancer screening ${ }^{[6]}$. It was initiated in 1987, before PSA testing had become a routine test. Thus, at the first two screening rounds in 1987 and 1990, only digital rectal examination was done. In the next two rounds 1993 and 1996, PSA testing was done parallel to rectal examination. Although the study was relatively small, with only 1494 men in the screening group from the start, the long follow-up has rendered a statistical power of the same magnitude as that presented in the first report from the PLCO trial ${ }^{[2]}$. The study, however, was designed for assessing the feasibility, cost-effectiveness and side-effects of screening rather than analyzing the impact on prostate cancer mortality. Furthermore, only half of the men diagnosed with prostate cancer in the screening group were treated with radiotherapy or surgery. When followed for fifteen years, no difference in prostate cancer mortality between the screening and control groups was seen. The lack of reduction in prostate cancer mortality, seen in the Norrkoping trial, may be the result of a low detection rate and skepticism towards radical prostatectomy and radiotherapy when the study started.

\section{Discussion}

The seemingly contradictory outcomes of these studies are to a great extent dependent on differences in set-up, screening intervals, PSA thresholds, and management of men with prostate cancer. There is no uniform way of organizing prostate cancer screening. The shorter the screening interval 14 and the lower the PSA threshold, the higher is the sensitivity, yielding a higher detection rate at the cost of risk for over-detection and over-treatment (Figure 1). Whereas the screening sensitivity at the Gothenburg Centre in the ERSPC trial was high, the screening sensitivity was intermediate at the rest of the ERSPC centers and in the PLCO and Quebec trials and the Stockholm and Norrkoping trials had a low sensitivity.

Whether or not a high detection rate is beneficial for men undergoing screening can only be determined by the men who, on the one hand, face the risk of being falsely assumed to have a lethal cancer, or undergo advanced treatment of an indolent tumor without malignant potential yet, on the other, gain the chance of preventing a curable tumor from developing into a serious cancer that may ultimately shorten life, the last months of which are ridden with anguish, loss of control of basic functions, and therapy-resistant pain.

\section{References}

[1] Bill-Axelson A, Holmberg L, Ruutu M, Garmo H, Stark JR, Busch C, Nordling S, Häggman M, Andersson SO, Bratell S, Spångberg A, Palmgren J, Steineck G, Adami HO, Johansson JE; SPCG-4 Investigators. Radical prostatectomy versus watchful waiting in early prostate cancer. N Engl J Med. 2011 May 5;364(18):1708-17.

[2] Andriole GL, Crawford ED, Grubb RL 3rd, Buys SS, Chia D, Church TR, et al. for the PLCO Project Team. Mortality results from a randomized prostate-cancer screening trial. N Engl J Med 2009;360:1310-19

[3] Schröder FH, Hugosson J, Roobol MJ, Tammela TL, Ciatto S, Nelen V, et al.: ERSPC Investigators. Screening and prostate-cancer mortality in a randomized European study. N Engl J Med 2009;360:1320-28

[4] Kjellman A, Akre O, Norming U, Törnblom M, Gustafsson O. 15-year follow-up of a population based prostate cancer screening study. J Urol. 2009;181:1615-21; discussion 1621.

[5] Labrie F, Candas B, Dupont A, Cusan L, Gomez JL, Suburu RE, et al. Screening decreases prostate cancer death: First analysis of the 1988 Quebec prospective randomized controlled trial. Prostate. 1999;38:83-91.

[6] Sandblom G, Varenhorst E, Rosell J, Löfman O, Carlsson P. Randomised prostate cancer screening trial: 20 year follow-up. BMJ. 2011 Mar 31;342:d1539. doi: 10.1136/bmj.d1539. PMID: 21454449

[7] US Preventive Services Task Force. Screening for Prostate Cancer: US Preventive Services Task Force recommendation statement: draft: Summary of recommendation and evidence. http://www.uspreventiveservicestaskforce.org/uspstf12/prostate/prostateart.htm [Accessed January 17, 2011]. 
[8] Ladjevardi S, Sandblom G, Berglund A, Varenhorst E. Tumour grade, treatment, and relative survival in a population-based cohort of men with potentially curable prostate cancer. Eur Urol. 2010 Apr;57(4):631-38

[9] Jonsson A, Aus G, Berterö C. Men's experience of their life situation when diagnosed with advanced prostate cancer. Eur J Oncol Nurs. 2009 Sep;13(4):268-73.

[10] Sandblom G, Carlsson P, Sennfält K, Varenhorst E. A population-based study of pain and quality of life during the year before death in men with prostate cancer. Br J Cancer. 2004 Mar 22;90(6):1163-68

[11] Howard K, Salkeld GP, Mann GJ, Patel MI, Cunich M, Pignone MP. The COMPASs study: Community Preference for Prostate cAncer Screening. Protocol for a quantitative preference study. BMJ Open 2012;2

[12] Andriole GL, Crawford ED, Grubb RL 3rd, Buys SS, Chia D, Church TR, Fouad MN, Isaacs C, Kvale PA, Reding DJ, Weissfeld JL, Yokochi LA, O'Brien B, Ragard LR, Clapp JD, Rathmell JM, Riley TL, Hsing AW, Izmirlian G, Pinsky PF, Kramer BS, Miller AB, Gohagan JK, Prorok PC; for the PLCO Project Team. Prostate Cancer Screening in the Randomized Prostate, Lung, Colorectal, and Ovarian Cancer Screening Trial: Mortality Results after 13 Years of Follow-up. Natl Cancer Inst. 2012 Jan 6. [Epub ahead of print]. PMID: 22228146

[13] Hugosson J, Carlsson S, Aus G, Bergdahl S, Khatami A, Lodding P, et al. Mortality results from the Göteborg randomized population-based prostate-cancer screening trial. Lancet Oncol2010;11:725-32

[14] Van Leeuwen PJ, Roobol MJ, Kranse R, Zappa M, Carlsson S, Bul M, Zhu X, Bangma CH, Schröder FH, Hugosson J. Towards an optimal interval for prostate cancer screening. Eur Urol. 2012 Jan;61(1):171-76. 